\title{
Stellar population in two star-forming regions
}

\author{
N. Azatyan, E. Nikoghosyan, H. Harutyunian, D. Baghdasaryan, and D. Andreasyan \\ Byurakan Astrophysical Observatory, 0213, Aragatsotn prov., Armenia
}

\begin{abstract}
Our research focuses on the stellar content of two star-forming regions. The first one is the molecular cloud which includes G45.12+0.13 and G45.07+0.13 UC HII regions around IRAS 19111+1048 and 19110+1045 sources, respectively. Based on infrared photometric data, we identified a rich stellar population, which includes 909 YSOs with different evolutionary stages. Among selected YSOs there are ZAMS objects. The second one is an elongated molecular cloud, which includes IRAS $05184+3635,05177+3636,05168+3634,05162+3639$, and $05156+3643$ sources. We identified 1224 candidates of YSOs in the molecular cloud with different evolutionary stages. Selected YSOs are mostly younger then $0.1 \mathrm{Myr}$. The distribution of selected YSOs in both star-forming regions shows that there are dense clusters in the vicinity of all IRAS sources.
\end{abstract}

Keywords: stars: pre-main sequence - stars: luminosity function-infrared: stars - radiative transfer

\section{Introduction}

Presently, it is generally recognized that the stellar content of our Galaxy forms in clusters located within cold $(\mathrm{T} \sim 10-30 \mathrm{~K})$ and dense $\left(\mathrm{n}>10^{3} \mathrm{~cm}^{-3}\right)$ giant molecular clouds (GMC) of the Galactic disk (e.g. Blitz, 1991, Lada \& Lada, 2003). Therefore, the main properties of the stellar population in the embedded young clusters, such as the density distribution, the age and age spread, the star formation efficiency (SFE), and the shape of the initial mass function (IMF) might be very well closely related to the physical properties and mass distribution of the progenitor structures in the parental cloud (e.g. McKee \& Ostriker, 2007). The development of sensitive, large-format imaging arrays at near- and mid-infrared (NIR and MIR), submillimeter, and radio wavelengths has made it possible to obtain statistically significant and complete sampling of young embedded clusters within molecular clouds.

In this paper, we present results of the NIR, MIR and far-infrared (FIR) study of two star-forming regions associated with IRAS $19110+1045$ and IRAS $19111+1048$ pair system and IRAS $05168+3634$ multiple system. The latter system contains four more IRAS sources (IRAS 05184+3635, IRAS 05177+3636, IRAS 05162+3639, and IRAS 05156+3643) embedded in the same molecular cloud (Azatyan, 2019). Figure 1 presents the color-composite images of both regions on Herschel images.

IRAS 19110+1045 and IRAS 19111+1048 objects are also referred to as G45.07+0.13 and G45.12+0.13 UCHII regions, respectively (Wood \& Churchwell, 1989). From the images obtained at 2 and $6 \mathrm{~cm}$ radio wavelengths, Wood \& Churchwell (1989) determined that the morphology of the ionized gas in the G45.07+0.13 region has a spherical shape, and in G45.12+0.13 - cometary. On the $1.3 \mathrm{~mm}$ continual images, G45.12+0.13 has an elliptical shape, elongated from north-east to south-west (Hernández-Hernández et al., 2014). Given their proximity in the plane of the sky and similar LSR velocities, Hunter et al. (1997) suggested that G45.12+0.13 and G45.07+0.13 lie at the same distance equal to $8.3 \mathrm{kpc}$. A more recent distance estimate, according to the trigonometric parallax, is $7.75 \pm 0.45 \mathrm{kpc}$ (Wu et al., 2019). We adopt a distance of $\sim 7.8 \mathrm{kpc}$ in our study. Many multi-wavelength studies suggest that both UC HII regions are sites of active massive star formation, at that IRAS 19111+1048 is a more advanced (Hunter et al., 1997, Vig et al., 2006). Both regions contain type I OH masers (Argon et al., 2000), but only IRAS $19110+1045$ contains $\mathrm{H}_{2} \mathrm{O}$ (Hofner \&

\footnotetext{
*nayazatyan@bao.sci.am, Corresponding author
} 

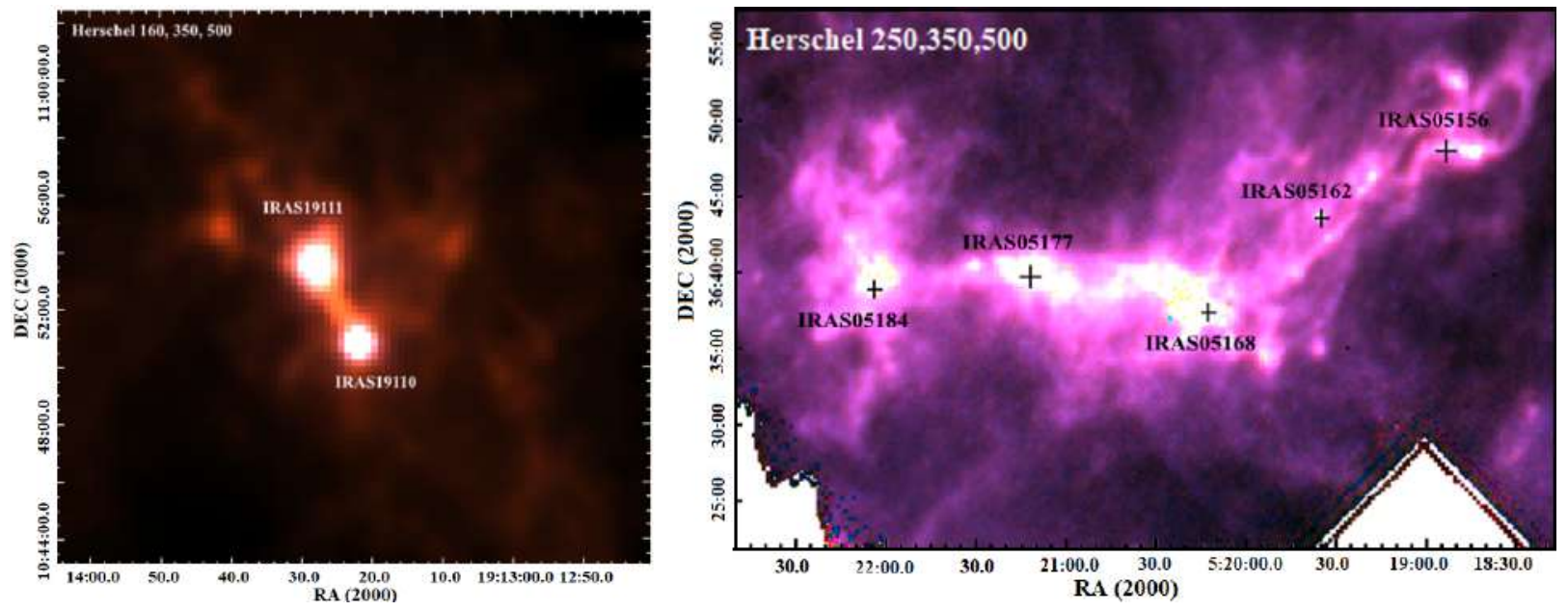

Figure 1. (left panel) Color-composite image of IRAS 19110+1045 and IRAS 19111+1048 pair system. (right panel) Color-composite image of IRAS 05168+3634 multiple system.

Churchwell, 1996) and methanol maser emissions (Breen et al., 2019, Hernández-Hernández et al., 2019). The NIR data, as well as high-resolution radio measurements, enabled Vig et al. (2006) and Rivera-Ingraham et al. (2010) to conclude that IRAS 19111+1048 region contains a larger number of ZAMS stars energizing compact and evolved HII region.

IRAS 05168+3634 is also known as Mol 9 in the catalog of Molinari et al. (1996). Zhang et al. (2005) have discovered a molecular outflow in this region. There have been various detections in the region: $\mathrm{H}_{2} \mathrm{O}$ maser emission (Zhang et al., 2005), $\mathrm{NH}_{3}$ maser emission (Molinari et al., 1996), CS emission (Bronfman et al., 1996), a new detection of $44 \mathrm{GHz} \mathrm{CH}_{3} \mathrm{OH}$ methanol maser emission (Fontani et al., 2010), the $\mathrm{SiO}(\mathrm{J}=2-1)$ line (Harju et al., 1998), the main lines at $1665 \mathrm{MHz}$ and $1667 \mathrm{MHz}$ $\mathrm{OH}$ maser (Ruiz-Velasco et al., 2016), and four ${ }^{13} \mathrm{CO}$ cores (Guan et al., 2008). IRAS $05184+3635$ and IRAS 05177+3636 are associated with dark clouds DOBASHI 4334 and 4326, respectively (Dobashi, 2011). IRAS $05162+3639$ is associated with the $\mathrm{H}_{2} \mathrm{O}$ maser (Sunada et al., 2007). A high propermotion star has been detected in the LSPM-NORTH catalog 0.35 arcmin from the IRAS $05156+3643$ (Lépine \& Shara, 2005) probably compatible with IRAS 05156+3643 within the error bars. The distance estimations of this multiple system are different. A kinematic distance was estimated of $6.08 \mathrm{kpc}$ (Molinari et al., 1996). The trigonometric parallax of IRAS $05168+3634$ corresponds to a distance of $1.88_{-0.17}^{+0.21} \mathrm{kpc}$ (Sakai et al., 2012). In Casoli et al. (1986), the distances of IRAS $05184+3635$ and IRAS $05177+3636$ were evaluated at the same $1.4 \mathrm{kpc}$ distance. Both distances were used for further study. The embedded stellar cluster in this region was detected in the NIR and MIR by various authors (Azatyan et al., 2016, Faustini et al., 2009, Kumar et al., 2006). Azatyan et al. (2016) revealed a bimodal cluster with 1.5 arcmin radius from geometric center of the cluster that does not coincide with IRAS $05168+3634$ source.

\section{Used data}

For our study, we used the data covering a wide range from NIR to FIR wavelengths. The first is the archival NIR photometric data in the $\mathrm{J}, \mathrm{H}$, and $\mathrm{K}$ bands of the Galactic Plane Survey DR6 (UKIDSS GPS, Lucas et al., 2008). Archival data of MIR observations were obtained from the Galactic Legacy Infrared Midplane Survey Extraordinaire (GLIMPSE, Churchwell et al., 2009), made with the Spitzer Infrared Array Camera (IRAC, Fazio et al., 2004). The four IRAC bands are centered at approximately 3.6, 4.5, 5.8, and $8.0 \mu \mathrm{m}$. We also used the Wide-field Infrared Survey Explorer (WISE, Wright et al., 2010) in 3.4, 4.6, 12, and $22 \mu \mathrm{m}$ bandpasses. We used FIR wavelengths, in the range 70-500 $\mu \mathrm{m}$, obtained by using the Photodetector Array Camera and Spectrometer (PACS, Poglitsch et al., 2010) and the Spectral and Photometric Imaging Receiver (SPIRE, Griffin et al., 2010) at the $3.5 \mathrm{~m}$ Herschel Space Observatory (Pilbratt et al., 2010). 

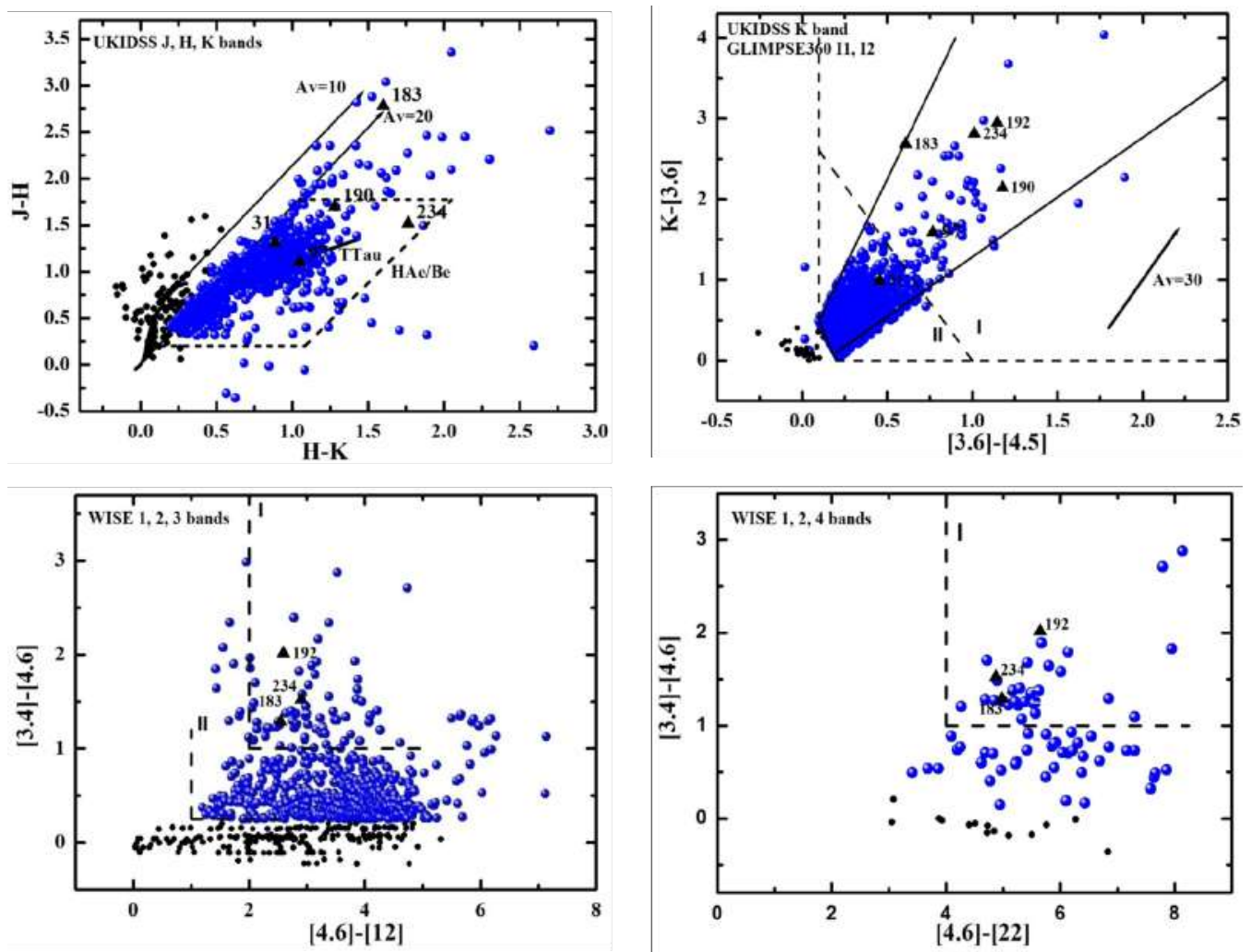

Figure 2. Color-color diagrams of the IRAS 05168+3634 multiple system. In the top left panel is shown the (J-H) vs. (H-K) diagram. In the top right panel the K-[3.6] vs. [3.6]-[4.5] diagram is presented. In bottom left and right panels are shown the [3.4]-[4.6] vs. [4.6]-[12] and [3.4]-[4.6] vs. [4.6]-[22] diagrams. The blue circles are selected YSOs and black circles are unclassified ones. IRAS and MSX sources are indicated by triangles and squares, respectively, and they are labeled as https://doi.org//10.26093/cds/vizier.36220038 catalog. For a detailed description of the references from which the locus of dwarf and giant stars, reddening vectors, and positions of different types of YSOs were obtained, see the text.

\section{Results and Discussions}

\subsection{Color-color diagrams}

For the selection of objects in both molecular clouds, we used the data of NIR, MIR, and FIR catalogs (see Section 2) within a radius of 6 arcmin concerning the geometric center of IRAS 19110+1045 and IRAS $19111+1048$ pair system and a radius of 24 arcmin concerning the conditionally selected IRAS $05168+3634$ source. We chose GPS UKIDSS-DR6 as the main catalog, and the other catalogs were cross-matched with it. The GPS UKIDSS-DR6 catalog for individual objects provides a probability (in percent) of being a star, galaxy, and noise; therefore, we selected objects with a probability of being noise $<50 \%$, and taking into account the completeness limit of UKIDSS survey in $\mathrm{K}$ band, the objects that have a measured magnitude of $K \geqslant 18.02$ were removed from the list. The MIR and FIR photometric catalogs were cross-matched with the GPS UKIDSS-DR6 catalog within $3 \sigma$ of combined error matching radius.

One of the most powerful tools for identifying YSO candidates via reddening and excess is their location on color-color diagrams. The investigated regions are quite large and there is a high probability of selecting objects that do not belong to the considered molecular clouds; therefore, we chose as YSOs those stars that are classified as objects with IR excess in at least two color-color diagrams to minimize the likelihood of choosing incorrectly. Since IRAS 19110+1045 and IRAS 19111+1048 pair system has two saturated areas in the MIR around the IRAS objects, therefore within those areas the selection of YSOs was based on only NIR color-color diagram.

Figure 2 (top left panel) shows the (J-H) versus (H-K) color-color diagram of IRAS $05168+3634$ where the solid and dashed curves represent the locus of the intrinsic colors of dwarf and giant stars, taken from Bessell \& Brett (1988) after being converted to the CIT system using the relations given 
Table 1. Properties of the cluster

\begin{tabular}{lcccccc}
\hline \hline IRAS & $\begin{array}{c}\alpha(2000) \\
(\mathrm{hh} \mathrm{mm} \mathrm{ss})\end{array}$ & $\begin{array}{c}\delta(2000) \\
(\mathrm{dd} \mathrm{mm} \mathrm{ss})\end{array}$ & $\begin{array}{c}\alpha(2000) \\
(\mathrm{hh} \mathrm{mm} \mathrm{ss})\end{array}$ & $\begin{array}{c}\delta(2000) \\
(\mathrm{dd} \mathrm{mm} \mathrm{ss})\end{array}$ & $\begin{array}{c}\text { Radius } \\
(\operatorname{arcmin})\end{array}$ & $\mathrm{N}$ \\
\hline$(1)$ & $(2)$ & $(3)$ & $(4)$ & $(5)$ & $(6)$ & $(7)$ \\
\hline $05184+3635$ & 052153.2 & +363820.4 & 052152.6 & +363907.1 & 2.5 & 52 \\
$05177+3636$ & 052109.4 & +363937.1 & 052102.8 & +363828.5 & 3.5 & 79 \\
$05168+3634$ & 052016.4 & +363718.7 & 052022.3 & +363733.9 & 3 & 57 \\
$05162+3639$ & 051938.4 & +364225.0 & 051938.4 & +364225.0 & 0.25 & 5 \\
$05156+3643$ & 051903.6 & +364615.7 & 051904.0 & +364802.0 & 2.8 & 47 \\
$19111+1048$ & 191327.8 & +105336.7 & 191327.3 & +105407.4 & 1.2 & 101 \\
$19110+1045$ & 191322.0 & +105054.0 & 191323.1 & +105051.9 & 0.8 & 44 \\
\hline
\end{tabular}

Notes. (1)-Name of clusters, (2),(3)-The coordinates of IRAS sources, (4),(5)-The coordinates of geometric centers of the clusters, (6)-The radius of each cluster according to YSOs surface density distribution, (7)Number of objects within refined radii.

by Carpenter (2001). The parallel solid lines drawn from the base and tip of the dwarf and giant loci, are the interstellar reddening vectors (Rieke \& Lebofsky, 1985). The locus of unreddened classical T Tauri stars (CTTSs) is taken from Meyer et al. (1997). The region bounded by the dashed lines where the pre-main sequence (PMS) stars with intermediate mass, i.e., Herbig Ae/Be stars are usually found (Hernández et al., 2005). For further study, we chose objects located to the right of reddening vectors that have a considerable, accurately expressed IR excess. Among the selected YSOs and objects within the reddening band of the MS and giant, we classified as Class I evolutionary stage objects those which have (J-K) $>3$ mag color index (Lada \& Adams, 1992) and located in the top right in the $(\mathrm{J}-\mathrm{H})$ versus $(\mathrm{H}-\mathrm{K})$ diagram. We used the same color-color diagram also for IRAS $19110+1045$ and IRAS $19111+1048$ pair system.

We used the K-[3.6] versus [3.6]-[4.5] color-color diagram for both star-forming regions. Figure 2 (top right panel) shows the K-[3.6] versus [3.6]-[4.5] color-color diagram of IRAS 05168+3634, where the diagonal lines outline the region of location of YSOs with both Class I and Class II evolutionary stages. The de-reddened colors are separated into Class I and II domains by the dashed line. Arrow shows the extinction vector (Flaherty et al., 2007). All the lines in the K-[3.6] versus [3.6]-[4.5] diagram are taken from Allen et al. (2007).

We also constructed two other color-color diagrams for both star-forming regions using the list of objects with good WISE detections, i.e., those possessing photometric uncertainty $<0.2 \mathrm{mag}$ in WISE bands. Figure 2 (lower left panel) shows the [3.4]-[4.6] versus [4.6]-[12] color-color diagram of IRAS $05168+3634$. As was mentioned for the previous color-color diagrams, the objects with different evolutionary stages are located in certain places in this diagram too (Koenig et al., 2012), i.e., Class I YSOs are the reddest objects, and are selected if their colors match [3.4]-[4.6] $>1.0$ and [4.6]-[12] $>2.0$. Class II YSOs are slightly less red objects and are selected with colors [3.4]-[4.6]- $\sigma([3.4]-[4.6])>0.25$ and [4.6]-[12]- $\sigma([4.6]-[12])>1.0$, where $\sigma(\ldots)$ indicates a combined photometric error, added in quadrature.

We can check the accuracy of previous classification of stars that possess photometric errors $<0.2 \mathrm{mag}$ in WISE band 4. Previously classified Class I sources were re-classified as Class II if [4.6]$[22]<4.0$ and Class II stars have been placed back in the unclassified pool if [3.4]-[12] $<-1.7 \times([12]-$ $[22])+4.3$ (Koenig et al., 2012). However, there are no incorrect selections in the pre-classified objects with 1, 2, 3 bands and it confirms the results obtained in the [3.4]-[4.6] versus [4.6]-[12] color-color diagram (Figure 2, lower right panel).

In total, we selected 1224 YSOs within a 24 arcmin radius concerning the conditionally selected IRAS $05168+3634$ source and 909 YSOs within a 6 arcmin radius of IRAS 19110+1045 and IRAS 19111+1048 system. Figure 3 shows the distribution of classified YSOs in both star-forming regions. Class I and Class II objects are indicated by filled red and blue circles, respectively. It can be seen that excluding the regions in the vicinity of the IRAS sources, all types of stellar objects are distributed relatively homogeneously in both molecular clouds. We also can see that in all cases, close to IRAS sources, the 

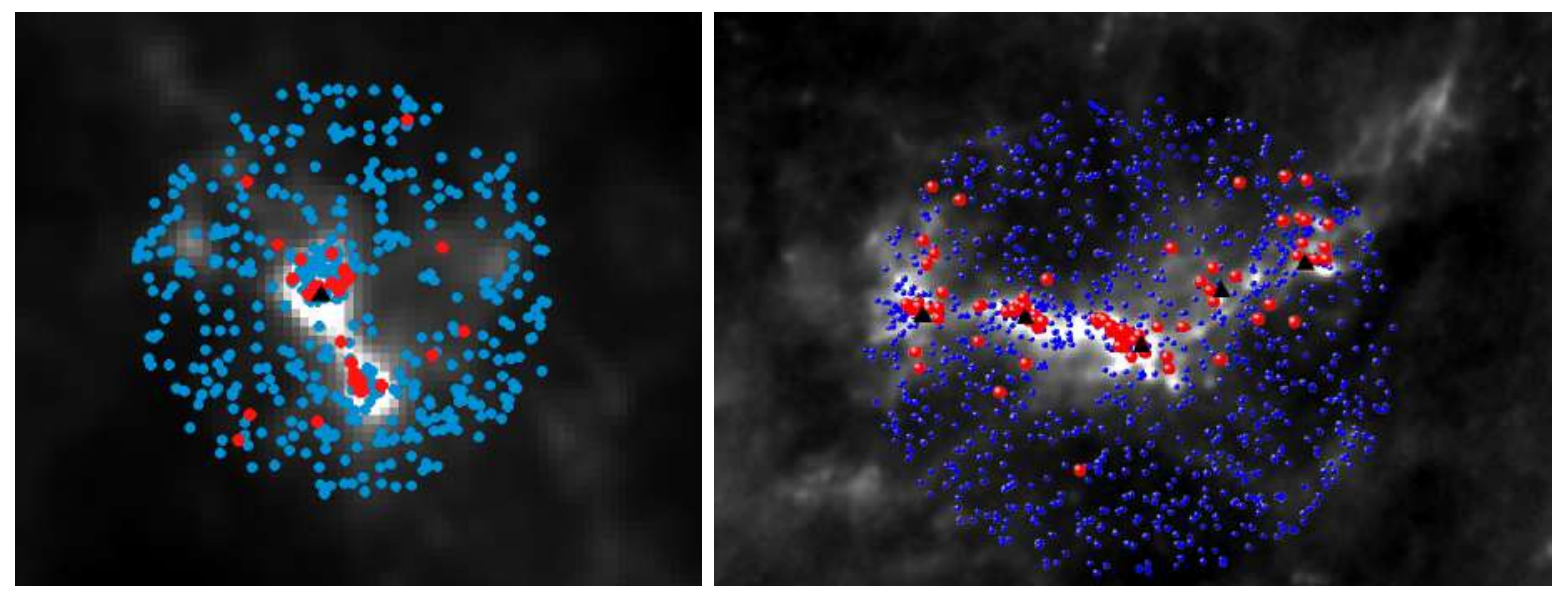

Figure 3. Distribution of YSOs in the regions on Herschel $500 \mu \mathrm{m}$ images. left panel presents the distribution of YSOs in IRAS 19110+1045 and IRAS 19111+1048 pair system and right panel - in IRAS 05168+3634 multiple system. Class I and Class II objects are indicated by filled red and blue circles, respectively. IRAS sources are indicated by black triangles.

selected YSOs form relatively dense concentrations or clusters.

Since the regions are quite large, further investigations were performed on concentration areas. For that purpose, we refined the radius of each cluster relative to their geometric centers based on the density distribution of selected YSOs. Table 1 presents the coordinates of IRAS sources in Cols. 2 and 3, the coordinates of geometric centers are in Cols. 4 and 5, and the radii based on stellar density distribution are in the Cols. 6. The numbers of objects within selected radii are in the last column. There is no evidence of a real concentration only around IRAS $05162+3639$. Therefore, the 0.25 arcmin value of radius given in Table 1 is conditional. So further studies were conducted for the 240 YSOs from IRAS 05168+3634 multiple system and 145 YSOs from IRAS 19110+1045 and IRAS 19111+1048 pair system; this total numbers of objects falls within the already-defined radii of the clusters.

\subsection{Color-magnitude diagrams}

The color-magnitude diagram is a useful tool for studying the nature of the stellar population within star-forming regions and for estimating its spectral types. The distribution of the identified YSOs in the $\mathrm{K}$ versus J-K color-magnitude diagrams are shown in Figure 4 with different symbols for each cluster. The zero age main sequence (ZAMS, thick solid curve) and PMS isochrones for ages 0.1, 0.5, and $5 \mathrm{Myr}$ (thin solid curves) are taken from Siess et al. (2000). We used the conversion table of Kenyon et al. (1994). Figure 4 left panel shows K versus J-K color-magnitude diagram for members of IRAS 19110+1045 (blue circles) and IRAS 19111+1048 (red circles) clusters. To correct $\mathrm{J}$ and K magnitudes of the selected YSOs in IRAS 19110+1045 and IRAS 19111+1048 clusters, we used $7.8 \mathrm{kpc}$ distance, and the average value of interstellar extinction $\mathrm{A}_{v}=13 \mathrm{mag}$ (see Section 3.3 ). According to the location of the stellar objects in the color-magnitude diagram, we can see that $\sim 80 \%$ of the objects from IRAS $19110+1045$ and IRAS $19111+1048$ cluster are located to the left of $1 \mathrm{Myr}$ isochrone. In general, the members of both clusters are brighter in the $\mathrm{K}$ band.

Figure 4 middle and right panels show $\mathrm{K}$ versus J-K color-magnitude diagrams for members of IRAS 05184+3635 (red circles), IRAS 05177+3636 (green circles), IRAS 05168+3634 (blue circles), IRAS 05162+3639 (yellow circles), and IRAS 05156+3643 (pink circles) clusters. The J and K photometry of the selected members are corrected for two different distances: 6.1 and $1.88 \mathrm{kpc}$, and estimated average $\mathrm{A}_{v}=4.5 \mathrm{mag}$ toward five IRAS sources (Azatyan, 2019). In general, the selected YSOs are distributed to the right of $0.1 \mathrm{Myr}$ isochrones, especially in the case of $6.1 \mathrm{kpc}$ distance, and this distribution confirms that they are YSOs. Only a few identified YSOs are located to the left (black circles) of ZAMS.

The López-Chico \& Salas (2007) correction can be used to obtain a lower limit of the stellar masses in both star-forming regions with excess vector, which has directions such as presented in Figure 4. Since all detected objects in IRAS 19110+1045 and IRAS 19111+1048 clusters are brighter in the K- 

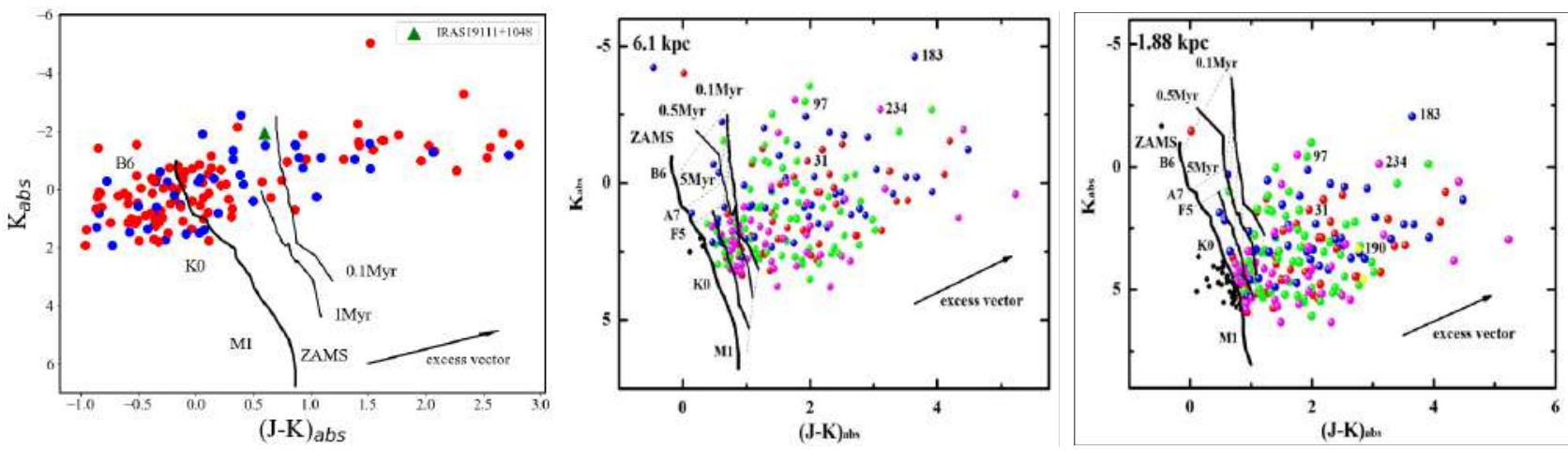

Figure 4. K vs. (J-K) color-magnitude diagrams for the identified YSOs in clusters. (left panel) The positions of the YSOs from IRAS 19110+1045 (blue circles) and IRAS 19111+1048 (red circles) pair system. IRAS 19111+1048 source is indicated by a green triangle and it is labelled. (middle and right panels) The positions of the YSOs from IRAS $05168+3634$ multiple system for distances 6.1 and $1.88 \mathrm{kpc}$. The objects belonging to different clusters are shown as follows: IRAS 05184+3635 (red circles), IRAS 05177+3636 (green circles), IRAS 05168+3634 (blue circles), IRAS 05162+3639 (yellow circles), and IRAS 05156+3643 (pink circles). Black circles indicate objects considered members of clusters, but in the color-magnitude diagram they are located to the left of ZAMS. The IRAS and MSX sources are labeled as https://doi.org//10.26093/ cds/vizier. 36220038 catalog. The arrow indicates the average slope of NIR excesses caused by disks around YSOs, as determined by López-Chico \& Salas (2007).

band than the estimation of lower mass obtained of $1.4 \mathrm{M}_{\odot}$ value. A large distance and interstellar extinction can play a crucial role in this result. At a distance of $1.88 \mathrm{kpc}$, approximately $80 \%$ of the total content of the clusters in IRAS $05168+3634$ multiple system has $<1$ solar mass, and the remaining $\backsim 20 \%$ objects have $1-3$ solar masses. At a distance of $6.1 \mathrm{kpc}$, approximately $20 \%$ of the total content of the clusters has $<1$ solar mass, about $70 \%$ objects have $1-3$ solar masses.

\subsection{SED analysis}

In order to learn about the evolutionary stages of the YSOs that have measured magnitudes in longer wavelengths, we constructed their spectral energy distributions (SEDs) and fitted them with the radiative transfer models of Robitaille et al. (2007). The SEDs are constructed for the members of clusters in both star-forming regions. This procedure was done using wavelengths ranging from $1.1 \mu \mathrm{m}$ to $500 \mu \mathrm{m}$. The SED fitting tool was carried out using both distance estimations for IRAS $05168+3634$ multiple system: 1.88 and $6.1 \mathrm{kpc}$. We used the ranges of $\mathrm{A}_{v}$ and the distances of $1-40 \mathrm{mag}$, and 5.5$6.5 \mathrm{kpc}$ and $1.6-2 \mathrm{kpc}$, respectively. For IRAS $19110+1045$ and IRAS $19111+1048$ clusters, we used the ranges of $\mathrm{A}_{v}$ and the distances of 10-100 mag and 6.5-9.5 kpc, respectively.

Table 2 presents the parameters of IRAS sources estimated by SED fitting tool. For the clusters of IRAS $05168+3634$ system at distances of 1.88 and $6.1 \mathrm{kpc}$, objects associated with IRAS sources can be classified as middle-mass YSOs. We applied SED fitting tool for 120 YSOs out of 240 selected ones in the clusters of IRAS $05168+3634$ multiple system. We could obtained the parameters of only IRAS $19111+1048$ source which is satisfy the conditions to create an UC HII region. For IRAS $19110+1045$ source, we were unable to identify an object that could satisfy the conditions of central star(s) of this UC HII region. We carried out SED fitting tool only for 29 YSOs out of 145 selected ones in IRAS 19110+1045 and IRAS 19111+1048 clusters due to the saturation in MIR ranges and therefore the lack of photometric data. According to the SED fitting tool, the average value of interstellar extinction is equal to $\mathrm{A}_{v}=13 \mathrm{mag}$ in this pair system, which was used for the correction of $\mathrm{J}$ and $\mathrm{K}$ photometry in $\mathrm{K}$ versus $\mathrm{J}-\mathrm{K}$ color-magnitude diagram (see Section 3.2).

\section{Conclusions}

The search, identification, and classification of the young stellar population in the molecular clouds surrounding IRAS 19111+1048 and IRAS 19110+1045 pair system and IRAS 05168+3634 multiple 
Table 2. Parameters of IRAS sources derived from Robitaille et al. (2007) models SED fitting.

\begin{tabular}{lccccccc}
\hline \hline IRAS & $\begin{array}{c}\text { Distance } \\
(\mathrm{kpc})\end{array}$ & $\begin{array}{c}\mathrm{A}_{v} \\
(\mathrm{mag})\end{array}$ & $\begin{array}{c}\text { Stellar age } \\
(\mathrm{Log})(\mathrm{yr})\end{array}$ & $\begin{array}{c}\text { Stellar mass } \\
\left(\mathrm{M}_{\odot}\right)\end{array}$ & $\begin{array}{c}\text { Temperature } \\
(\mathrm{K})\end{array}$ & $\begin{array}{c}\mathrm{L}_{\text {Total }} \\
(\mathrm{Log})\left(\mathrm{L}_{\odot}\right)\end{array}$ & $\begin{array}{c}\text { Class } \\
(1)\end{array}$ \\
\hline$(2)$ & $(3)$ & $(4)$ & $(5)$ & $(6)$ & $(7)$ & $(8)$ \\
\hline $05184+3635$ & 1.88 & $12.1 \pm 2.0$ & $6.6 \pm 5.9$ & $3.1 \pm 0.6$ & $12589 \pm 2000$ & $1.9 \pm 1.5$ & $\mathrm{II} / \mathrm{I}$ \\
$05184+3635$ & 6.1 & $6.9 \pm 0.3$ & $5.9 \pm 5.8$ & $6.8 \pm 0.7$ & $12589 \pm 8000$ & $3.0 \pm 2.8$ & $\mathrm{II} / \mathrm{I}$ \\
$05177+3636$ & 1.88 & $4.4 \pm 1.8$ & $5.8 \pm 5.9$ & $2.6 \pm 1.3$ & $6309 \pm 3981$ & $2.4 \pm 2.5$ & $\mathrm{I}$ \\
$05177+3636$ & 6.1 & $3.0 \pm 1.0$ & $4.1 \pm 4.0$ & $5.5 \pm 0.9$ & $3981 \pm 630$ & $2.6 \pm 2.0$ & $\mathrm{I}$ \\
$05168+3634$ & 1.88 & $15.3 \pm 1.0$ & $6.0 \pm 5.4$ & $5.1 \pm 0.3$ & $15848 \pm 1584$ & $2.8 \pm 2.0$ & $\mathrm{I}$ \\
$05168+3634$ & 6.1 & $6.9 \pm 2.9$ & $5.9 \pm 5.5$ & $7.1 \pm 1.1$ & $19952 \pm 6309$ & $3.4 \pm 2.7$ & $\mathrm{I}$ \\
$05162+3639.1$ & 1.88 & $6.3 \pm 3.1$ & $4.8 \pm 4.7$ & $1.0 \pm 0.4$ & $3981 \pm 501$ & $1.2 \pm 0.5$ & $\mathrm{I}$ \\
$05162+3639.1$ & 6.1 & $7.8 \pm 2.5$ & $4.2 \pm 4.0$ & $3.1 \pm 0.6$ & $3981 \pm 630$ & $2.1 \pm 1.4$ & $\mathrm{I}$ \\
$05162+3639.2$ & 1.88 & $34.6 \pm 3.0$ & $5.9 \pm 5.2$ & $4.5 \pm 0.3$ & $7943 \pm 1000$ & $2.2 \pm 1.8$ & $\mathrm{I}$ \\
$05162+3639.2$ & 6.1 & $19.5 \pm 3.8$ & $6.0 \pm 5.2$ & $5.4 \pm 0.2$ & $15848 \pm 1000$ & $2.8 \pm 1.7$ & $\mathrm{I}$ \\
$05156+3643$ & 1.88 & $2.1 \pm 0.8$ & $6.2 \pm 6.3$ & $3.4 \pm 3.6$ & $12589 \pm 7943$ & $3.2 \pm 3.4$ & $\mathrm{I}$ \\
$05156+3643$ & 6.1 & $1.4 \pm 1.9$ & $3.8 \pm 2.7$ & $2.9 \pm 1.2$ & $3981 \pm 316$ & $2.3 \pm 0.4$ & $\mathrm{I}$ \\
$19111+1048$ & 7.8 & $12.0 \pm 4.6$ & $6.40 \pm 6.07$ & $9.4 \pm 4.3$ & $22928 \pm 10771$ & $3.8 \pm 3.6$ & $\mathrm{II}$ \\
\hline
\end{tabular}

Notes. (1)-Name of IRAS source, (2)-Distance, (3)-(7)-the weighted means and the standard deviations of parameters obtained by SED fitting tool for all models with best $\chi^{2}-\chi_{\text {best }}^{2}<3 \mathrm{~N}$, (8) - classification of YSOs.

system made it possible to obtain the following results: (i) Census of the young stellar population have been obtained in both regions, (ii) the distribution of selected YSOs shows clear concentrations in the vicinity of IRAS sources, (iii) in IRAS $05168+3634$ multiple system unlike the Class II objects, the Class I objects are located mainly in vicinity of IRAS sources, (iv) selected YSOs in IRAS $05168+3634$ multiple system are mostly younger then 0.1 Myr, ( $v$ ) among selected YSOs in IRAS 19111+1048 and IRAS $19110+1045$ pair system there are ZAMS objects.

\section{Acknowledgements}

The authors thanks to the organizers for the opportunity to take a part in International conference 'Astronomical Surveys and Big Data 2'

\section{References}

Allen L., et al., 2007, Protostars and Planets V, pp 361-376

Argon A. L., Reid M. J., Menten K. M., 2000, ApJS, 129, 159

Azatyan N. M., 2019, A\&A, 622, A38

Azatyan N. M., Nikoghosyan E. H., Khachatryan K. G., 2016, Astrophysics, 59, 339

Bessell M. S., Brett J. M., 1988, PASP, 100, 1134

Blitz L., 1991, in Lada C. J., Kylafis N. D., eds, NATO Advanced Science Institutes (ASI) Series C Vol. 342, NATO Advanced Science Institutes (ASI) Series C. p. 3

Breen S. L., Contreras Y., Dawson J. R., Ellingsen S. P., Voronkov M. A., McCarthy T. P., 2019, MNRAS, 484, 5072

Bronfman L., Nyman L.-A., May J., 1996, A\&AS, 115, 81

Carpenter J. M., 2001, AJ, 121, 2851

Casoli F., Dupraz C., Gerin M., Combes F., Boulanger F., 1986, A\&A, 169, 281

Churchwell E., et al., 2009, PASP, 121, 213

Dobashi K., 2011, PASJ, 63, S1

Egan M. P., et al., 2003, VizieR Online Data Catalog, 5114

Faustini F., Molinari S., Testi L., Brand J., 2009, A\&A, 503, 801

Fazio G. G., et al., 2004, ApJS, 154, 10 
Fish V. L., Reid M. J., Wilner D. J., Churchwell E., 2003, ApJ, 587, 701

Flaherty K. M., Pipher J. L., Megeath S. T., Winston E. M., Gutermuth R. A., Muzerolle J., Allen L. E., Fazio G. G., 2007, ApJ, 663,1069

Fontani F., Cesaroni R., Furuya R. S., 2010, A\&A, 517, A56

Griffin M. J., et al., 2010, A\&A, 518, L3

Guan X., Wu Y., Ju B., 2008, MNRAS, 391, 869

Han X. H., Zhou J. J., Wang J. Z., Esimbek J., Zhang J. S., Wang N., 2015, A\&A, 576, A131

Harju J., Lehtinen K., Booth R. S., Zinchenko I., 1998, A\&AS, 132, 211

Hernández-Hernández V., Zapata L., Kurtz S., Garay G., 2014, ApJ, 786, 38

Hernández-Hernández V., Kurtz S., Kalenskii S., Golysheva P., Garay G., Zapata L., Bergman P., 2019, AJ, 158, 18

Hernández J., Calvet N., Hartmann L., Briceño C., Sicilia-Aguilar A., Berlind P., 2005, AJ, 129, 856

Hofner P., Churchwell E., 1996, A\&AS, 120, 283

Hunter T. R., Phillips T. G., Menten K. M., 1997, ApJ, 478, 283

Kenyon S. J., Gomez M., Marzke R. O., Hartmann L., 1994, AJ, 108, 251

Koenig X. P., Leisawitz D. T., Benford D. J., Rebull L. M., Padgett D. L., Assef R. J., 2012, ApJ, 744, 130

Kumar M. S. N., Keto E., Clerkin E., 2006, A\&A, 449, 1033

Lada C. J., Adams F. C., 1992, ApJ, 393, 278

Lada C. J., Lada E. A., 2003, ARA\&A, 41, 57

Lépine S., Shara M. M., 2005, AJ, 129, 1483

López-Chico T., Salas L., 2007, Rev. Mex. Astron. Astrofis., 43, 155

Lucas P. W., et al., 2008, MNRAS, 391, 136

McKee C. F., Ostriker E. C., 2007, ARA\&A, 45, 565

Meyer M. R., Calvet N., Hillenbrand L. A., 1997, AJ, 114, 288

Molinari S., Brand J., Cesaroni R., Palla F., 1996, A\&A, 308, 573

Molinari S., Brand J., Cesaroni R., Palla F., Palumbo G. G. C., 1998, A\&A, 336, 339

Pilbratt G. L., et al., 2010, A\&A, 518, L1

Poglitsch A., et al., 2010, A\&A, 518, L2

Price S. D., Egan M. P., Carey S. J., Mizuno D. R., Kuchar T. A., 2001, AJ, 121, 2819

Rieke G. H., Lebofsky M. J., 1985, ApJ, 288, 618

Rivera-Ingraham A., et al., 2010, ApJ, 723, 915

Robitaille T. P., Whitney B. A., Indebetouw R., Wood K., 2007, ApJS, 169, 328

Ruiz-Velasco A. E., Felli D., Migenes V., Wiggins B. K., 2016, ApJ, 822, 101

Sakai N., Honma M., Nakanishi H., Sakanoue H., Kurayama T., Shibata K. M., Shizugami M., 2012, PASJ, 64, 108

Siess L., Dufour E., Forestini M., 2000, A\&A, 358, 593

Sunada K., Nakazato T., Ikeda N., Hongo S., Kitamura Y., Yang J., 2007, PASJ, 59, 1185

Vig S., Ghosh S. K., Kulkarni V. K., Ojha D. K., Verma R. P., 2006, ApJ, 637, 400

Wood D. O. S., Churchwell E., 1989, ApJS, 69, 831

Wright E. L., et al., 2010, AJ, 140, 1868

Wu Y. W., et al., 2019, ApJ, 874, 94

Zhang Q., Hunter T. R., Brand J., Sridharan T. K., Cesaroni R., Molinari S., Wang J., Kramer M., 2005, ApJ, 625,864 